\title{
Resolution of migraine attacks: sleep and the recovery phase
}

\author{
JN BLAU \\ From the National Hospitals for Nervous Diseases, Queen Square, and Maida Vale, and the City of \\ London Migraine Clinic, London
}

SUMMARY A study of 50 migraineurs has confirmed previous observations that sleep is a common way of ending the headache phase. 14 subjects could shorten attacks by going to sleep during the day for an average of $2 \frac{1}{2}$ hours. Different methods used by patients to aid falling asleep are described. 47 of the 50 subjects had symptoms after the headache had gone-here called the recovery phase which can double the length of individual attacks. It is suggested that prodromata, some symptoms of the headache and recovery phases, as well as the therapeutic effect of sleep, indicate that migraine is primarily a neurological rather than a vascular disorder.

This inquiry was stimulated by a patient saying that she could shorten and end her migraine attacks by making herself sleep for two hours. Perhaps other patients had found similar or alternative methods to abbreviate attacks that could be more widely used in treatment. Furthermore, techniques which resolve migraine episodes could illuminate the underlying, still unknown "migraine process" that initiates and maintains individual attacks.

When asking patients how their attacks ended, a range of symptoms that persisted after the headache had resolved became apparent. Although patients often state that after an attack they feel "washed out", these symptoms have gained scant attention, even by Liveing, ${ }^{1}$ one of the few who annotated how attacks terminated in individual patients.

\section{Patients and methods}

50 subjects with uncomplicated migraine were questioned: 29 were patients referred by their personal physician to a neurological or migraine clinic; 21 were colleagues and friends, 11 being medically qualified. Having ascertained that they had migraine as previously defined, ${ }^{2}$ each was asked how their migraine ended and if they had tried or found ways of shortening attacks, then how they felt after the headache had gone, followed by specific questions about feeling tired, yawning, mental, physical, alimentary and urinary symptoms, and their duration. The answers

Address for reprint requests: $\mathrm{Dr}$ JN Blau, The National Hospital for Nervous Diseases, Queen Square, London WC1N 3BG, UK.

Received 14 October 1981\}

Accepted 1 November 1981 to questions about the same symptoms before and during the headache phase were also sought and recorded. Ages ranged from 21 to 64 years with an average of 42 years. 35 were female and 15 male. 34 had prodromes ${ }^{3}$ and 16 a classical aura.

\section{Results}

HOW HEADACHES RESOLVED

Slowly during the day: 18 subjects found that their headache decreased imperceptibly during the day"it just drifts away", or "I realised it had disappeared during the morning or afternoon". Rapidly by day: eight individuals noted a rapid decline of pain estimated between $\frac{1}{4}$ and 1 hour; in four of these, vomiting preceded pain relief. Sleep at night: 28 of the 50 subjects noted relief from headache after a night's rest. Many retired early if the pain was moderate or severe, if it was opportune to do so. A few added that such a sleep seemed particularly deep. Sleep by day: 14 subjects ended some attacks by going to bed during daylight hours, always excluding light by drawing the curtains or putting the head under the bed clothes. Additional methods to attain sleep will be detailed in the next section. The duration of day time sleep required to end the headache varied from $\frac{1}{2}$ to 6 hours, the average being $2 \frac{1}{2}$ hours. One physician found that "a siesta of $\frac{3}{4}$ hour sufficed". Another consultant physician, if his headache was severe while at hospital, would sit and snooze for 10-15 minutes in a dark quiet place until his pain diminished sufficiently to complete the day's work and then go home, early to bed. Of the 50 subjects, 29 felt that one of the above modes pre- 
Table Methods of shortening attacks-32 patients

\begin{tabular}{|c|c|c|}
\hline $\begin{array}{l}\text { Unaided sleep } \\
\text { Retiring early (1) } \\
\text { Sleep by day (2) }\end{array}$ & $\begin{array}{l}\text { Sleep aided by heat or cold } \\
\text { Hot bath (1) } \\
\text { Hot water bottle to painful site (1) } \\
\text { Cold flannel to neck (1) }\end{array}$ & $\begin{array}{l}\text { Sleep and medication } \\
\text { Aspirin (3) } \\
\text { Paracetamol (2) } \\
\text { "Distalgesic" (1) } \\
\text { "Migril" (1) } \\
\text { Diazepam (1) }\end{array}$ \\
\hline $\begin{array}{l}\text { Sleep, medication and heat (4) or cold (2) } \\
\text { Analgaesic (4) } \\
\text { "Cafergot" (1) } \\
\text { Metoclopramide (1) }\end{array}$ & $\begin{array}{l}\text { Food, with or without medication } \\
\text { Black coffee (1) } \\
\text { Tea (1) } \\
\text { Cooked meal (1) } \\
\text { Cooked meal and analgaesic (1) } \\
\text { Tea, cake and mefenamic acid (1) } \\
\text { Hot soup to induce vomiting (1) }\end{array}$ & $\begin{array}{l}\text { Other methods } \\
\text { Hot bath and analgaesic (1) } \\
\text { Ergot inhaler (1) } \\
\text { "Migril" (2) } \\
\text { Paracetamol and fresh air (1) } \\
\text { Deep breathing, analgaesic and sleep (1) }\end{array}$ \\
\hline
\end{tabular}

dominantly ended attacks, 19 by two methods, one by three and one in four different ways. A few observed that the pattern of ending attacks changed as they grew older, but all agreed that no method was invariably efficacious.

\section{METHODS OF ABBREVIATING OR REDUCING SEVERITY OF ATTACKS}

18 subjects said that nothing helped to shorten attacks which had to take their own course. This did not inhibit patients taking analgesics, anti-migraine preparations or retiring earlier than usual to try and end their attacks. Of the remaining 32, the following methods (table) at times, but by no means invariably, reduced the length or the severity of attacks, or both. Some subjects used a number of techniques.

A number of subjects talked about trying to go to sleep. One "switched off mentally", and another "reduced the work load" implicating a conscious effort to relax or go to sleep but they could not specify how they achieved their result.

\section{SYMPTOMS AFTER HEADACHE HAD} DISAPPEARED-RECOVERY PHASE

Only three patients in this series were totally symptom-free once the headache ceased. The other 47 had mood variations, muscular weakness, abnormal appetite, yawning, tiredness and changes in fluid balance. 31 of these 47 had multiple symptoms: "tired and washed out" was a common expression, for feeling mentally and physically below normal. Although the symptoms lasted from 1 hour to 4 days in individuals, "the rest of the day", "the following morning" or "the whole of the next day" were usual descriptions. All patients commented that the post-headache symptoms were more marked after moderate or severe attacks.

\section{MOOD CHANGES}

Elevation of mood from slightly euphoric to "on top of the world with a desire to spring-clean the house" or "high and really happy" occurred in eight. More frequent, in 28, was a lowering or mood and intellect, interfering with figure work, concentrating, alertness, unproductive (a research scientist), inclined to finish the morning clinic at $\mathbf{1 1 . 3 0}$ am instead of $1 \mathrm{pm}$ (a consultant physician), dopey, irritable, low, lifeless (a journalist), muddled, inattentive, sluggish, not fully alert and distant were terms used. In most instances routine work was possible but more arduous or creative mental activities were difficult, or not tackled.

\section{PHYSICAL AND MUSCULAR SYMPTOMS}

Physical tiredness featured in 26 and muscular weakness in 27 . In extreme instances aching muscles were compared with an influenzal illness. Others lacked normal energy- "just get meals but not a lot of housework" (a 35-year-old housewife), or "ordinary daily activities but not playing football or badminton" (a 26-year-old bank official).

\section{APPETITE AND FLUID BALANCE}

Restricted food intake was noted by 16 subjects, especially intolerance to rich, fatty or spicy foods. Bland meals that could be taken included boiled potatoes, steamed fish, a sandwich and "carbohydrate in general" (a doctor), toast, biscuits were common. Two subjects specifically desired dry toast and Marmite and six expressed a need for sweet foods or sweets, similar to the cravings experienced during the prodromal phase. ${ }^{3}$ Seven passed more than usual amounts of urine, two less and four were thirsty.

\section{YAWNING AND TIRED}

Four yawned excessively, one without feeling tired, while in the other three excessive tiredness was evident.

OTHER FEATURES OF ATTACKS IN THIS

SERIES

Prodromal symptoms occurred in 34 of the 50 patients, many having more than one symptom. Attacks were 


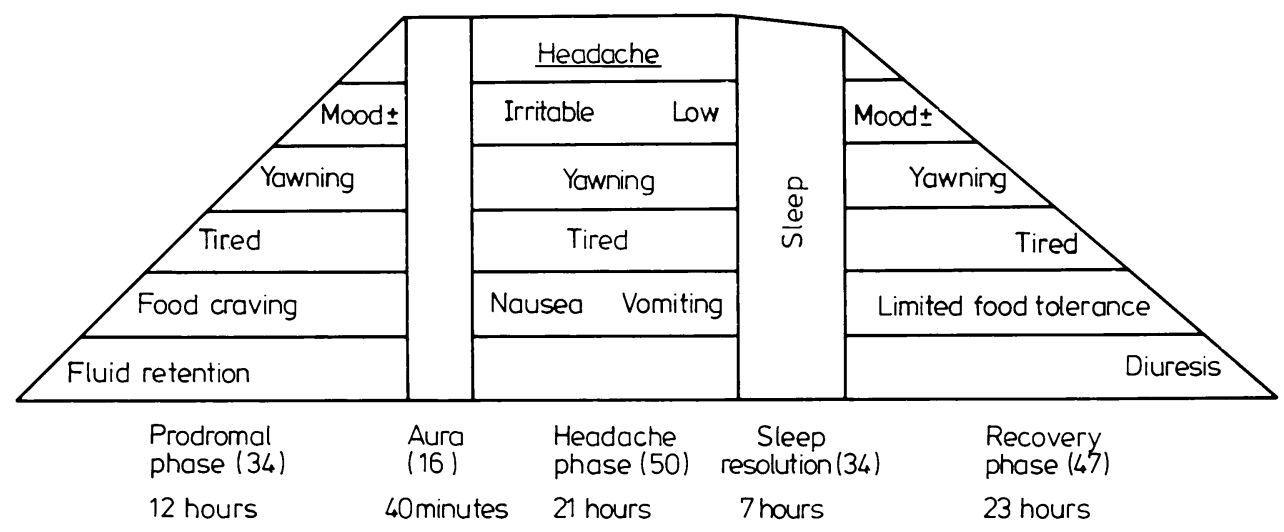

Figure A diagram illustrating phases, average duration and symptoms in migraine attacks from 50 subjects with uncomplicated migraine.

heralded by mood elevation (13), depression (12), yawning (15), undue tiredness (10), anorexia or nausea (6), constipation (3), loose bowel action (2), increased desire for food, usually sweets or chocolate (8), fluid retention (3), feeling unduly cold (2), sneezing (1) and sweating (2).

The duration of prodromal symptoms before an aura, if one occurred, or before the onset of headache ranged from 1 to 48 hours: the average in 34 subjects was 12 hours. The high incidence of prodromes may be due to including some subjects from a previous series. ${ }^{3}$

Aura 16 patients had an aura, visual disturbances for 15-30 minutes (12), dysaesthesiae or dysphasia for $\frac{1}{2}-2$ hours (3), or brain-stem features of basilar migraine lasting $1 \frac{1}{2}$ hours (1). The average duration in this group was $\mathbf{4 0}$ minutes.

Duration of the headache phase Headaches lasted from 4 hours to $3 \frac{1}{2}$ days, the average in the 50 cases being 21 hours.

Sleep The range of sleep duration during which the headache disappeared was $\frac{1}{2}-6$ hours, if patients slept by day, or 8-12 hours during the night, depending on whether they retired early or at the normal time - a mean of 7 hours.

SUMMATION OF TIMING AND A DIAGRAM

The duration of the various phases is based on each patient's estimate, liable to be influenced by the most recent attacks in some, or recollections of more severe attacks in others. Hence the times are approximate. If a patient said that a particular phase took 4-6 hours, then 5 hours was taken, or $\frac{1}{2}-1$ day then 18 hours was accepted. On this basis a diagram (fig) was drawn.

\section{Discussion}

The observation that sleep resolved migraine attacks was recorded 100 years ago by Liveing ${ }^{1}$ who mentioned a number of his famous contemporaries whose attacks ended in this way, including DuBois Reymond, the discoverer of the electrical nature of the neural impulse. More recently Dr Marcia Wilkinson has maintained that an anti-emetic followed by an analgaesic enabled patients to go to sleep, and it was the process of sleeping that relieved the headache most efficiently. She has proven her point by a statistical analysis of 310 patients treated at the City Migraine Clinic in London. ${ }^{4}$ Confirmation comes from a headache clinic in New York: Coddon (personal communication) gave intravenous chlorpromazine, diazepam and then sodium amylobarbitone. He found that 3-4 hours sleep was essential to end migraine attacks.

The therapeutic implications for migraineurs are self-evident: they must become acquainted with the various known methods of stopping attacks and experiment on themselves to discover which method or combination helps them. It could prove beneficial to those patients whose attacks resolve during sleep to study how to get to sleep as soon as possible after the onset of symptoms, even by day. Darkness, lying down (although a few prefer to sit), heat or cold, anti-emetics, analgaesics, anti-migraine preparations, singly or in combination may assist falling asleep. Perhaps the pharmaceutical companies should reconsider the inclusion of caffeine in their antimigraine compound preparations, another point made by Dr Wilkinson and supported by this study.

The theoretical significance of sleep in relation to 
migraine needs appraisal. Normal sleep is thought to have a restorative function. ${ }^{5}$ Sleep after a migraine attack could therefore be a non-specific response to stress, pain, vomiting or lack of food. Alternatively somnolence, yawning and sleep may be integral to the altered physiology in migraine, a notion supported by these observations: attacks can be precipitated by lack of sleep, becoming excessively tired or sleeping too long; yawning and tiredness are prodromal symptoms that may continue during, as well as after, the headache phase.

The sleep-waking cycle is affected by hypothalamic disturbances. Other cyclical changes implicating the hypothalamus shown by the migraineurs in this series were mood variations, fluid retention, diuresis, hyperphagia, nausea and vomiting followed by restricted food tolerance. Hence neuro-endocrinological studies, particularly of the hypothalamus suggested by Herberg, ${ }^{6}$ could be rewarding. A potential mechanism for these vegetative symptoms has been postulated recently. ${ }^{7}$

Finally, turning to the underlying migraine pathogenesis - is it vascular or neural? Discussing this problem, Gowers ${ }^{8}$ wrote 100 years ago, "the vascular system is in a special way under the influence of the cerebral centres. An emotional blush and the pallor of fear are conspicuous examples of this fact. It is at least as easy to conceive that the vascular changes are the result of the disturbance in the sensory centres, or are the effect of associated derangement of the vasomotor centres, as it is to consider that the vascular condition is the primary change".

If Gowers' analogy is valid, then the vascular changes in migraine would be secondary to focal cerebral metabolic or primary neural disturbances. Recent experimental evidence indicates that vascular changes follow alterations in brain metabolism. For example, Sokoloff ${ }^{9}$ has demonstrated that functional activity in the central nervous system regulates local glucose metabolism and within seconds the regional microcirculation is secondarily adjusted to the local metabolic demands.

In migraine research the clinical evidence must be paramount. The presence of prodromal and recovery symptoms suggest that neurological disturbances form part of the underlying "migraine process" which still remains elusive. Although our aim is to abolish the condition by prevention, we are not yet in that happy position and must try to stop attacks as quickly as possible. Nevertheless, this study supports an old observation, the importance of sleep, which by itself indicates that further neurological research in relation to migraine would be worth while.

\section{References}

${ }^{1}$ Liveing E. On megrim, sick-headache, and some allied disorders. London: Churchill, 1873.

${ }^{2}$ Blau JN. Migraine Research. Br Med J 1971;ii:751-7.

${ }^{3}$ Blau JN. Migraine prodromes separated from the aura; complete migraine. Br J Med 1980;ii :658-60.

${ }^{4}$ Wilkinson M, Williams K, Leyton M. Observations on the treatment of an acute attack of migraine. Res Clin Stud Headache. Basel: Karger, 1978;6:141-6.

${ }^{5}$ Adam K, Oswald I. Sleep is for tissue restoration. $J$ R Coll Phys 1967-8;11:376-88.

${ }^{6}$ Herberg LJ. The hypothalamus and the aetiology of migraine. In: Smith R, ed. Background to Migraine. London: Heinemann Medical, 1967:96-112.

${ }^{7}$ Willoughby JO. The pathophysiology of vegetative symptoms in migraine. Lancet 1981,ii:445-6.

${ }^{8}$ Gowers WR. A manual of diseases of the nervous system. London: Churchill, 1888:790.

${ }^{9}$ Sokoloff L. Local cerebral energy metabolism: its relationship to local functional activity and blood flow. In: Elliott K, O'Connor M, eds. Cerebral vascular muscle and its control. Ciba Foundation Symposium 56 (New Series). Amsterdam: Elsevier, 1978:171-91. 